\title{
Clinical Airway Management: An Illustrated Case-Based Approach
}

\author{
D. John Doyle, Basem Abdelmalak (Editors). Cambridge University Press, 2017. Price \\ \$99.99 (hardcover), 370 pages. ISBN 978-1-316-60135-8
}

\author{
Gemma Malpas, MBChB, FANZCA
}

Received: 19 September 2017/ Accepted: 3 October 2017/Published online: 10 October 2017

(C) Canadian Anesthesiologists' Society 2017

Since the birth of cardiopulmonary resuscitation and the creation of its "ABCs," the management of "A," the airway, has understandably become a focus of education and research. Clinical Airway Management: An Illustrated Case-Based Approach covers virtually all aspects of managing the adult airway. This comprehensive textbook is neatly divided into seven main sections: Introduction to the Difficult Airway, Basic Airway Management, The Anticipated Difficult Airway, The Unanticipated Difficult Airway, Lower Airway Management, Special Situations and Airway Emergencies, and When the Airway Goes Bad. The text is beautifully formatted into short paragraphs and sections, making it not only highly readable but also a useful reference book and educational resource for all involved in airway management. The chapter style is consistent throughout the book despite considerable diversity in the authors' areas of expertise, geography, and specialty. The text's clinical focus and case-based approach helps keep the reader engaged throughout. The rich illustrations that accompany the text, despite being mostly black and white, complement the narrative well.

The first section of this book, Introduction to the Difficult Airway, comprises an overview of airway assessment and introduces the concept of the difficult airway. With the rapidly evolving literature on airway management, any next edition could ideally provide updates on the latest published airway guidelines and algorithms. The chapter, Pharmacology of Airway Management: A Minimal Synopsis, provides a clear, brief overview of essential medications required for

G. Malpas, MBChB, FANZCA ( $₫)$

Dalhousie University, Halifax, NS, Canada airway management, although additional clinical context would be helpful.

The next section, Basic Airway Management, begins with an introduction to airway management during procedural sedation. It then proceeds with management strategies needed as the depth of anesthesia progresses from light sedation to general anesthesia. All the basic tools of airway management, including supraglottic airway devices, direct laryngoscopes, and video laryngoscopes, are addressed in this section. With such a wide range of devices on the market, the section does well to describe the optimum techniques and uses for these devices, summarized by world-renowned experts in those fields.

The third section of this textbook focuses on The Anticipated Difficult Airway. The opening chapter describes a simple approach to awake bronchoscopic intubation and the circumstances that necessitate its use. The approach presented leans heavily on the use of nerve blocks to provide local anesthesia despite studies suggesting that most clinicians use topical airway anesthesia for this purpose. Perhaps with the anticipated success of this clinical textbook, the subject could be addressed in more detail in a future edition. The remainder of this section does a wonderful job of addressing many challenging airway scenarios. Each chapter describes different approaches to the management of a wide range of clinical scenarios in which a difficult airway would likely be encountered. The case-based presentation and discussion approach is a useful way to tackle these challenging airway topics.

The fourth section of the textbook is titled The Unanticipated Difficult Airway. The opening chapter addresses the unanticipated anterior larynx. It seems that the main purpose of this chapter is to describe 
airway maneuvers that could be employed during laryngoscopy to improve the glottic view. It also describes the steps to take when initial attempts at airway management have failed. The remaining four chapters address strategies for airway management in the obese patient and the parturient who require general anesthesia as well as an emergency surgical airway and the management of patients with accidental decannulation of a fresh tracheostomy. Despite addressing topics of relevance to the anesthesia provider, these chapters could easily be relocated to the sections on The Anticipated Difficult Airway and Airway Emergencies and Special Situations.

Unlike some other airway texts that have focused on glottic and supraglottic aspects of airway management, this textbook comprehensively addresses management issues from the upper airway to the lower airway. The fifth section, Lower Airway Management, describes techniques for managing tracheal and bronchial pathology - airway anatomy that is often feared by many anesthesia providers. Although management is sometimes controversial, airway pathology in these areas can result in significant morbidity and high mortality rates if managed by inexperienced practitioners. Hence, including this anatomical area in an airway textbook is of great value. These chapters provide an easy-to-read, safe, concise approach to airway management in challenging situations.

The remainder of the book goes on to include the sections Special Situations and Airway Emergencies and When the Airway Goes Bad. These sections comprise an interesting arrangement of case reports of various difficult airways and emergency situations, including an expanding neck hematoma, cranial facial trauma, and airway bleeds, to name just a few. Complementing earlier chapters that detailed technical management strategies, this case-based section is a useful reinforcement of core principles, although for some cases unusual management strategies are suggested.

In conclusion, Clinical Airway Management, An Illustrated Case-Based Approach is a teaching tool with clinical application of airway techniques for many complex airway scenarios. It discusses real scenarios in a contemplative style, using both descriptive text and beautiful illustrations. Tailored primarily to the North American market, this book is an excellent resource for all anesthesia practitioners and their trainees.

Conflicts of interest None declared.

Editorial responsibility This submission was handled by Dr. Hilary P. Grocott, Editor-in-Chief, Canadian Journal of Anesthesia. 